\title{
EQUIPE DO \\ ESCRITÓRIO DE INTEGRAÇÃO
}

\section{EQUIPE 2012:}

Coordenador: Prof. Álvaro José Paiva de Almeida

Coordenador adjunto: Profa. Hilda Cotegipe Pellico

Secretária: Bruna Cristine de Oliveira Cabral

Arquiteto egresso: Alfredo Lanna Neto

Alunos (estagiários e extensionistas): Ana Luiza Sampaio Braga, Bárbara Beatriz Magalhães Fernandes, Fabiana Mata da Silva, Maria Lúcia Alves Luiz, Raphaela de Almeida Pereira, Rayane França Paes Queiroz, Rogério Guimarães Misk Filho, Tais Servano Pereira Fernandes, Wagner de Paula Amaral Filho

\section{EQUIPES 2000-2012:}

Coordenações: Prof. Álvaro José Paiva de Almeida (05/2012 - 06/2013); Prof. Roberto Eustáaquio dos Santos (08/2010 04/2012); Profa. Vanessa Borges Brasileiro (08/2009 - 07/2010); Prof. Alfio Conti (08/2007 - 07/2009); Profa. Margarete Maria de Araújo Silva (02/2002 - 07/2009); Prof. Luís Inácio Sampaio (1993 - 2001)

Professores no El: Alfio Conti, Alícia Duarte Penna, Álvaro José Paiva de Almeida, Clécio Magalhães do Vale, Hilda Cotegipe Pellico, Luiz Inácio Sampaio, Margarete Maria de Araújo Silva, Maria Elisa Baptista, Mauricio Leonard, Roberto Eustáaquio da Silva, Vanessa Borges Brasileiro, Willian Sidney.

Professores consultores / colaboradores da PUC Minas: Anivaldo Matias de Sousa (eng. eletricista), Bráulio de MagaIhães Santos (bolsista de pós-graduação), Ilídio Valentim Lobato (eng. civil), José Nonato (eng. Civil), Marianna Vieira Rodrigues Maciel (advogada), Mário Omar Soares (eng. civil), Nilton Abdala Filho (eng. eletricista), Regina Coelli (assistente social), Roberto Pereira da Silva (arq.), Rogério Palhares Zschaber de Araújo (arq.), Sávio Estolano Grossi (eng. eletricista), Wellington B. Calixto (calculista).

\section{Arquitetos egressos do Curso de Arquitetura e Urbanismo} da PUC Minas: Adriana Ribeiro de Gouveia, Adrienne Oliveira Lessa, Alam Cristiam Sena, Alfredo Lanna Neto, Ana Cecília Rodrigues Moreno, Breno Notini, Camila Veloso Duarte Nascimento, Danilo de Carvalho Botelho de Almeida, Débora Bastos Marani, Fabiana Pedrosa, Fabrício Campolina Pinto, Gabriel Velloso, Izabela Galletti de Azevedo, José Mário Barbosa Alves, Lidiana Antunes Velloso, Luciana Miglio Machado, Luis Felipe de Farias, Marcelo Palhares Santiago, Maria Paula Corrêa Silva, Marília Bueno, Marina Beatriz Tello Oliveira, Mateus Gouvea de 
Sousa, Matheus Melo, Melissa Mendes, Natália Batista BoteIho, Paula Brey-Gil Faria, Rafael Mantuano Netto, Regina Coeli de Oliveira, Rodrigo Belo Bueno, Talassa Carvalho Andrade, Tatiana Soledade Delfanti Melo, Thiago Machado Laje Moreira.

\section{Alunos do Curso de Arquitetura e Urbanismo da PUC Mi- nas (estagiários e extensionistas): Adélia Natali Churata} Urbano, Ademar Alves de Oliveira Júnior, Adilson Assis Cruz Junior, Adriany Lopes de Araújo, Ágda Hereen Resende de Oliveira, Alecsandra Santos Cunha, Alessandra Otoni, Alessandra Rocha de Barcelos, Alexandre de Salvo, Alexandre Lanza, Ana Beatriz Beraldo Rubens, Ana Carolina Sacco, Ana Cassia Moura, Ana Cláudia Mendes Pontes, Ana Flávia Barbosa, Ana Lúcia Paulinelli, Ana Luiza Sampaio Braga, André Marinho dos Anjos, Anna Thereza da Motta, Antônio Esteves do Nascimento Júnior, Antonio Silva, Bárbara Beatriz MagaIhães Fernandes, Bruna de Almeida Sampaio, Bruno Camelo Bagetti, Camila Santos de Resende Chaves, Carmem Pedrosa, Carolina Drumond Franco, Cassio Eduardo, Christiane Thrisctler, Christianne M. Lima, Clara Estella Gómez Sánches, Clarice Lacerda Tibúrcio, Claudio Barcelos, Cristiane Martins de Oliveira, Cristiano Teodoro, Daniela Barbosa, Daniela Rabelo, Daniele Constantino, Daniele Vieira, Débora Maria Ramos, Denise Silveira, Eduardo Eugênio Fantini Zica, Eduardo Henrique Costa Lara, Eliete Alves dos Santos, Érico de Oliveira e Silva, Fabiana Cristina Costa e Silva, Fabiana Mata da Silva, Fábio Etelvino de Jesus Caetano, Fabrícia Borges, Faride Angélica, Felipe Palmer Caldeira Parreiras de Faria, Fabrícia Teixeira de Souza, Fernanda Glaciery Teixeira Ribas, Fernanda M Lopes, Fernanda Moreira, Fernanda Marcelly Pereira Almeida, Fernanda Mingote Colares Luz, Fernanda Takahashi, Fernando Abreu Fortes, Filipe Ioldionol Francisco Bandeira, Gabriela da Cunha Morais, Gisele Amanda Ferreira Othero, Gisele Figueiredo Gomes, Gidalfo Cabral Lopes, Giselle Oliveira Mascarenhas, Gustavo André, Gustavo Antônio Sotero Alves, Gustavo Perdigão, Isla de França, Israel Silveira Domingues, Ivone Pereira Castro, Janice Campos Vasconcelos, Janice Pereira, Jacqueline Sávia Noronha de Lima, Jéssika Estephanny Guimarães Marques, Jonas, Josiana Santos Neves, Julia Borges Pires Ferreira, Julia Hallack Sansão, Julia Rocha Araújo, Júlia Salgado Lins, Juliana Barreiros, Juliana Torres, Juliane Martins, Juliano Yuri de Oliveira Veloso, Júlio Fernandes, Junia Salomão Federman, Junio Gabriel Pereira Machado, Karla Valladares, Kátia Carvalho, Kátia Pettersen, Kleber Mendes Prodígios, Laís Grossi de Oliveira, Larissa Vilela Pena Siqueira, Leonardo Bicalho Pollizzi, Lisandra Mara Silva, Livia Turano Mota, Luana Maira Silva, Lucas Gazola, Lucas Henrique Marinho Costa, Lucas Pinheiro, Luciano Monteiro, Lucimar Moreira Carvalho, Ludmilla Amaral, Luíza Alana Fontes Moraes Silveira de Souza, Luiza Reis e Lima, Marcelo Dalmaso, Luíza Soares Silva Romeiro, Marcela Meira Machado, Marcelo Duarte Borges Caixeta, Marco Antônio Souza Borges Netto, Maria Cecília Andrade Lodi, Maria Helena Brandini Ferraz, Maria Lúcia Alves Luiz, Marina Silva dos Santos, Mariana Batista Mendes Rocha, Mariana Cançado Juste, Mariana Cardoso Araújo, Mariana Fernandes Balsamão Guimarães Diniz, Marina Oliveira Vasconcelos, Mario Antonio Ferrari 
Felisberto, Mateus Porto Hermeto, Matteo Gentilim, Mayson Tayrone Queiroz dos Santos, Natália Cardoso, Natália Welter, Nathana Miranda da Cunha, Nicole Portugal, Patrícia Colen, Patricia Marangoni Pimenta, Patrícia Monteiro de Castro, Paula Rodrigues Lotti Novaes, Pedro Henrique Barbosa Ribeiro, Priscila Musa, Querlis Silva, Rafaela Cristina de Paula Melo, Raimer Leal Lopes, Raíssa Santos Martins, Raphaela de Almeida Pereira, Raquel Drummond, Raquel Furtado Martins de Paula, Raquel Meneses, Rayane França Paes Queiroz, Rejane Coutinho Drumond, Renata Carazza Silveira, Renata de Oliveira Duarte, Renata Pietra, Renata Rodrigues Taranto, Ricardo Nelmann, Rogério Guimarães Misk Filho, Rosana Mendonça, Rúbia Nascimento, Rubiam Gomes de Moraes, Samantha Furbino, Sandra Mara, Sirlei Ferreira Lopes, Soraia Aparecida Farias, Stela Araujo, Tadeu Vinicius de França, Taís Servano Pereira Fernandes, Taísa Teixeira Campos, Tatiana Monteiro Ramos, Tatiane Pardini Valério, Thiago Rosa de Oliveira, Tiago Castelo Branco, Tiago Minoro Kato, Vanessa Taveira de Souza, Vinícius Barbosa Passos, Viviane Santos Rabelo, Wagner de Paula Amaral Filho, Wanderson Lopes Ávila de Oliveira, Yuri Hallen Reis Pacheco.

Secretárias do Escritório de Integração: Bruna Cristine de Oliveira Cabral, Kátia Patrícia Macedo, Bertha Judith Schwertner.

Colaboradores externos (consultores / parceiros): Antônio Carlos Martins Calixto (eng. civil), Antônia de Pádua (Presidente da UEMP - União Estadual por Moradia Popular), Carlos M. Teixeira (Arquitetos Sem Fronteiras), Cenir Aparecida da Silva (Mestre de Obras do Curso Construção e Cidadania), Claudenice R. Lopes (Pastoral da Rua),

ComPac (Conselho Municipal do Patrimônio Ambiental e Cultural de Contagem), Enice Fonseca Nahas (Secretária Municipal de Educação e Cultura da Prefeitura de Contagem), Flávio Mourão Agostini (Arquitetos Sem Fronteiras), Gustavo Melo, Jozânia Miguel Chaves (psicóloga), José A. Rodrigues, Júlia Carolina de Cunha (Núcleo de Memória da Prefeitura de Contagem), Liane N. Born (Rua Viva), Luciana Miglio (Arquitetos Sem Fronteiras), Maria Cristina Bove (Pastoral de Rua), Maura Aparecida Pereira de Matos (Pastoral de Rua), Mauricio Campolina Libânio (sociólogo), Mônica Santiago (odontologia), Mucio Tosta Gonçalves, Noêmia Rosana de Andrade (Núcleo de Memória da Prefeitura de Contagem), Sandra Regina Silva (pesquisadora).

\section{Agentes comunitários:}

Adão André de Souza, Anete Alves dos Santos Cabral, Ciro de Miranda, Eliom José de Souza, João Alves dos Santos, Leandro Silva de Assis do Nascimento, Luiz Ronaldo de Paulo Melo, Marlene Marques da Silva, Rosemary Expedita Oliveira, Sheila Mendes de Souza, Vanderlei Batista Dias. 

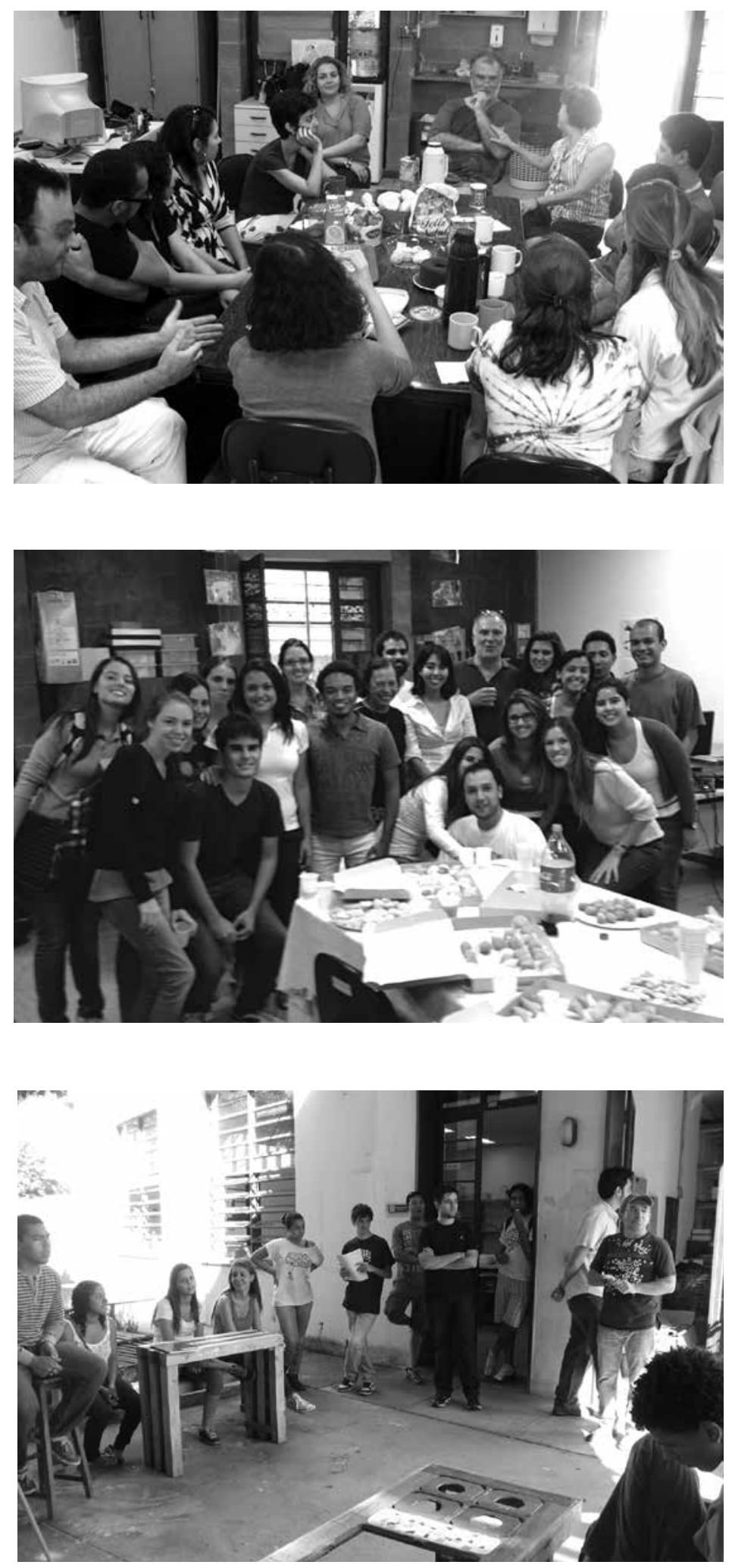

Cadernos de Arquitetura e Urbanismo, v.19, n.24+25, 2012 

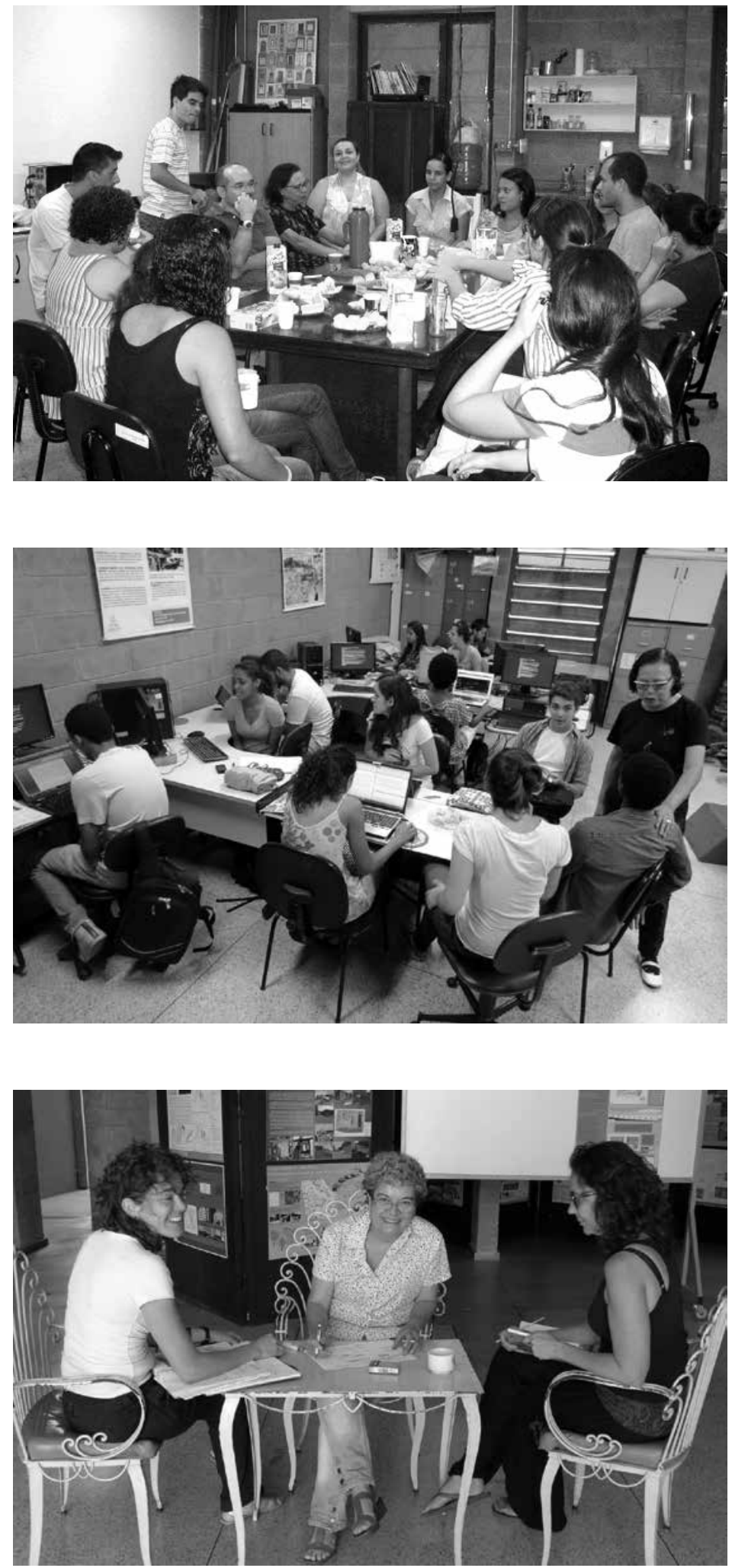

Cadernos de Arquitetura e Urbanismo, v.19, n.24+25, 2012 